\title{
TITLE:
}

\section{Classification of spinel structures based on first-principles cluster expansion analysis}

\section{$\operatorname{AUTHOR}(\mathrm{S})$ :}

Seko, Atsuto; Oba, Fumiyasu; Tanaka, Isao

\section{CITATION:}

Seko, Atsuto ...[et al]. Classification of spinel structures based on first-principles cluster expansion analysis. PHYSICAL REVIEW B 2010, 81(5): 054114.

ISSUE DATE:

2010

URL:

http://hdl.handle.net/2433/148380

RIGHT:

(C) 2010 The American Physical Society 
PHYSICAL REVIEW B 81, 054114 (2010)

\title{
Classification of spinel structures based on first-principles cluster expansion analysis
}

\author{
Atsuto Seko, ${ }^{1, *}$ Fumiyasu Oba, ${ }^{2}$ and Isao Tanaka ${ }^{2,3}$ \\ ${ }^{1}$ Pioneering Research Unit for Next Generation, Kyoto University, Kyoto 606-8501, Japan \\ ${ }^{2}$ Department of Materials Science and Engineering, Kyoto University, Kyoto 606-8501, Japan \\ ${ }^{3}$ Nanostructures Research Laboratory, Japan Fine Ceramics Center, Atsuta, Nagoya 456-8587, Japan \\ (Received 4 November 2009; revised manuscript received 31 January 2010; published 23 February 2010)
}

\begin{abstract}
We examine general trends of the ground-state structure and the order-disorder behavior in spinel oxides. We first determine the cation ordering in the ground state and investigate the temperature dependence of the cation distribution in IV-II spinel oxides by combining calculations based on the density-functional theory with the cluster-expansion method. We predict that $\mathrm{Mg}_{2} \mathrm{SnO}_{4}, \mathrm{Zn}_{2} \mathrm{SnO}_{4}$, and $\mathrm{Zn}_{2} \mathrm{TiO}_{4}$ have the inverse configuration while $\mathrm{Mg}_{2} \mathrm{TiO}_{4}$ has the normal configuration. On the basis of the results for IV-II spinel oxides and those for II-III spinel oxides, the spinels are then classified. The ground-state structure and order-disorder behavior can be classified using the parameters evaluated from the cluster expansion analysis.
\end{abstract}

DOI: 10.1103/PhysRevB.81.054114

PACS number(s): 81.30.Hd, 61.50.Ah, 81.30.Bx, 64.60.Cn

\section{INTRODUCTION}

Spinel is the famous mineral $\mathrm{MgAl}_{2} \mathrm{O}_{4} \cdot A B_{2} \mathrm{O}_{4}$ oxides often have the spinel structure. II-III spinel oxides with divalent cation $A$ and trivalent cation $B$ and IV-II spinel oxides with quadrivalent cation $A$ and divalent cation $B$ make up the majority of spinel oxides. In the spinel structure, oxygen atoms basically form a face-centered cubic (fcc) closedpacked sublattice. There are two kinds of cation sites in the spinel structure, which are tetrahedral fourfold-coordinated and octahedral sixfold-coordinated sites. The number of octahedral sites is double that of tetrahedral sites. When all of the octahedral sites are occupied by cation $B$, the spinel is called normal. When both cations $A$ and $B$ evenly occupy the octahedral sites, the spinel is called inverse. The normal and inverse spinels are generally expressed using the formulas $A\left[B_{2}\right] \mathrm{O}_{4}$ and $B[A B] \mathrm{O}_{4}$, respectively, where the square brackets designate the octahedral sites. Because inverse spinel has configurational degrees of freedom in the octahedral sites, cation ordering can be seen. The cation distribution is generally expressed using the degree of inversion $x$, which is defined as the fraction of cations $B$ in the tetrahedral sites or that of cations $A$ in the octahedral sites. The spinel with the degree of inversion $x$ is described by the formula $A_{1-x} B_{x}\left[A_{x} B_{2-x}\right] \mathrm{O}_{4}$.

It is important to understand the cation ordering and distribution in spinels because the physical properties of spinels depend not only on the chemical composition but also on their distribution. Many experiments have been performed to determine the cation distribution in a variety of compounds with the spinel structure for over seventy years. The trend of the experimental cation distribution in 172 spinel oxides was categorized with a success rate of $98 \%$ using a structural map, in which the spinel oxides are sorted by the sum of $s$ and $p$ nonlocal pseudopotential orbital radii of cations $A$ and $B .{ }^{1}$ The cation distribution depends heavily on atomic size and its electronegativity. In addition to the atomic characteristics, the cation distribution depends on temperature. As a matter of fact, it is difficult to determine the ground-state cation distribution experimentally. For metal oxides with high melting temperatures, thermal equilibrium conditions are generally very difficult to attain at low temperatures due to very low diffusion rates. For instance, the structure of $\mathrm{MgGa}_{2} \mathrm{O}_{4}$ reported from experiments has a wide range of $x$ values from 0.67 to $1 .^{2-6}$

Density-functional theory (DFT) calculations enable us to examine the cation ordering and distribution that are unknown or that are difficult to determine by experiments. Using DFT calculations, relative stabilities between the normal and disorder inverse configurations of 18 spinel oxides were systematically investigated. ${ }^{7}$ However, it is prohibitively expensive to calculate the energies of a large number of different configurations by DFT. The cluster expansion (CE) method $^{8-10}$ is a powerful tool for the evaluation of the configurational energies with the accuracy of the DFT calculations. Previously, we predicted the cation orderings and their temperature dependences in II-III spinel oxides $A B_{2} \mathrm{O}_{4}(A$ $=\mathrm{Mg}, \mathrm{Zn}$ and $B=\mathrm{Al}, \mathrm{Ga}, \mathrm{In}$ ) by combining DFT calculations with the CE method. ${ }^{11,12}$ In those studies, the cation orderings with the lowest energy of the six spinels were successfully reproduced. In addition, several types of order-disorder behaviors were recognized. The normal spinels have a unique order-disorder behavior from the ordered normal (ON) state to the disordered state, which can be described by the degree of inversion $x$ with the long-range nature. This order-disorder behavior is generally recognized as a nonconvergent one, in which the degree of inversion changes continuously without a phase transition. The inverse spinels have two kinds of order-disorder behavior. One is an orderdisorder behavior from the ordered inverse (OI) state to the disordered inverse (DI) state, which can be described by a long-range order parameter on the octahedral sites. The order-disorder behavior from the OI state to the DI state is a first-order transition. A second-order transition is ruled out by the Landau symmetry rules in the change from the OI state to the DI state. The DI state can be stabilized by the configurational entropy associated with the possibility of exchanging cations in the octahedral sites. The other is an order-disorder behavior from the DI state to the disordered state, which is described by the long-range degree of inversion $x$ as well as the order-disorder behavior in the normal spinel. This order-disorder behavior is generally recognized as a nonconvergent one. The disordered state is stabilized by 


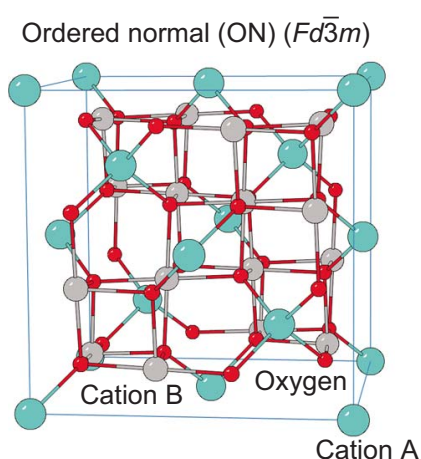

Ordered inverse (OI) $(P 4,22)$

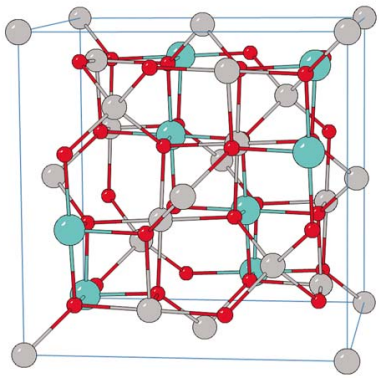

Disordered inverse (DI) $(F d \overline{3} m)$ Disordered-disordered (DD) $(F d \overline{3} m)$
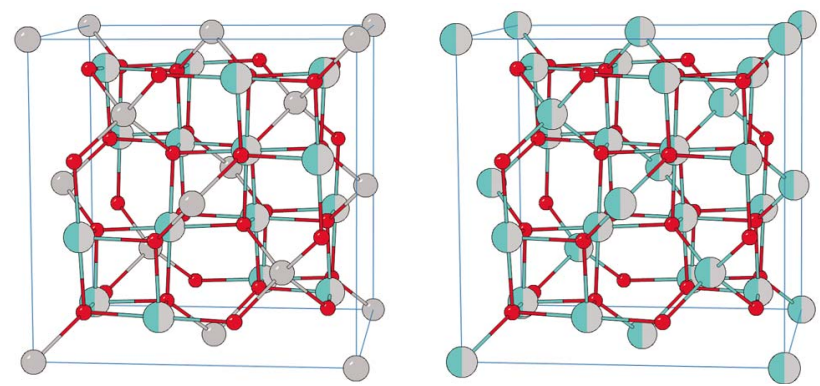

FIG. 1. (Color online) Four possible spinel states considered in the present study. The illustrated cation ordering of the OI state corresponds to the experimental crystal structures of $\mathrm{Mg}_{2} \mathrm{TiO}_{4}$ and $\mathrm{Zn}_{2} \mathrm{TiO}_{4}$ and the predicted crystal structures of $\mathrm{MgGa}_{2} \mathrm{O}_{4}$ and $\mathrm{MgIn}_{2} \mathrm{O}_{4}$.

the configurational entropy due to the exchange of cations in both tetrahedral and octahedral sites. This will hereafter be called the disordered-disordered (DD) state in order to distinguish it from the DI state. The four possible spinel states are drawn in Fig. 1.

In this paper, we first examine the cation ordering at zero temperature and the temperature dependence of the cation distribution in four kinds of IV-II spinel oxides, $\mathrm{Mg}_{2} \mathrm{TiO}_{4}$, $\mathrm{Zn}_{2} \mathrm{TiO}_{4}, \mathrm{Mg}_{2} \mathrm{SnO}_{4}$, and $\mathrm{Zn}_{2} \mathrm{SnO}_{4}$, systematically. The four oxides are experimentally known to be inverse spinels. ${ }^{13-16}$ It was also observed that the low-temperature forms of $\mathrm{Mg}_{2} \mathrm{TiO}_{4}$ and $\mathrm{Zn}_{2} \mathrm{TiO}_{4}$ exhibit cation ordering with the space group of $\mathrm{P}_{1} 22$, as shown in Fig. $1 .{ }^{15}$ The cation orderings in $\mathrm{Mg}_{2} \mathrm{SnO}_{4}$ and $\mathrm{Zn}_{2} \mathrm{SnO}_{4}$ are unknown. The configurational energy is evaluated by combining DFT calculations and the $\mathrm{CE}$ method. Combining them with the Monte Carlo (MC) simulation, the temperature dependence of the cation distribution is examined. On the basis of the results for IV-II spinel oxides in the present study and those for II-III spinel oxides in the previous study, ${ }^{12}$ general trends of the groundstate structure and the order-disorder behavior in spinel oxides are discussed. The ground-state structures can be satisfactorily classified using the parameters obtained by the firstprinciples $\mathrm{CE}$ analysis. Finally, an interesting order-disorder behavior in $\mathrm{Mg}_{2} \mathrm{TiO}_{4}$ is described.

\section{METHODOLOGY}

\section{A. Cluster-expansion method}

The CE method gives an effective representation of the configurational energy $E$. In a binary system, the configura- tional energy is expressed using pseudospin configuration variable $\sigma_{i}$ for lattice site $i$ as

$$
\begin{aligned}
E & =V_{0}+\sum_{i} V_{i} \sigma_{i}+\sum_{i, j} V_{i j} \sigma_{i} \sigma_{j}+\sum_{i, j, k} V_{i j k} \sigma_{i} \sigma_{j} \sigma_{k}+\cdots \\
& =\sum_{\alpha} V_{\alpha} \cdot \varphi_{\alpha},
\end{aligned}
$$

where $V_{\alpha}$ and $\varphi_{\alpha}$ are called the effective cluster interaction (ECI) and correlation function of cluster $\alpha$, respectively. Any thermodynamic functions and physical properties dependent on configurations, such as vibrational free energy, volume, bulk modulus, band gap, and critical temperature associated with the internal degrees of freedom, can be similarly expressed as the form of Eq. (1). A principal objective is to evaluate unknown ECIs as precisely as the configurational properties can be reconstructed within the accuracy of DFT calculations with $m$ truncated clusters. When total energies of $N(\geq m)$ ordered structures are prepared from DFT calculations, $m$ ECIs can be determined in the framework of the structure inversion method..$^{9,17}$ In this framework, the ECIs are generally determined by fitting a truncated form of Eq. (1) to DFT energies using the least-squares technique.

The appropriate selection of clusters and structures to DFT calculations (DFT structures) is crucial for the accuracy and the efficiency of the CE. In general, a set of clusters that minimizes the cross-validation $(\mathrm{CV})$ score $^{18,19}$ is searched for from the pool of candidate clusters using a general minimization algorithm. ${ }^{20}$ The leave-one-out $\mathrm{CV}$ score is defined as

$$
(\mathrm{CV})^{2}=\frac{1}{N} \sum_{i=1}^{N}\left(E_{i}-\hat{E}_{(i)}\right)^{2},
$$

where $E_{i}$ and $\hat{E}_{(i)}$ are the DFT energy of structure $i$ and the energy of structure $i$ predicted from a CE constructed from $(N-1)$ structures without structure $i$, respectively.

Since the CV score is evaluated using given input DFT structures, the accuracy for structures far from the inputs is generally lower than that for structures near the inputs. Therefore, the quality of the CE constructed from input DFT structures by minimizing the $\mathrm{CV}$ score should be validated using additional out-of-sample structures. Then, the CE should be improved using the additional out-of-sample structures. The additional structures should be chosen to cover as much of the configurational space as possible in order to reduce the $\mathrm{CE}$ error over the whole range of configurations. ${ }^{21}$ Therefore, the additional structures are chosen so as to significantly decrease the variance of the CE energy over the whole range of configurations.

The mean variance of the CE energy for all structures in the population is expressed as ${ }^{21}$

$$
\left\langle\operatorname{Var}\left[E_{\mathrm{CE}}(i)\right]\right\rangle=\left\{\operatorname{tr}\left[\left(X^{\mathrm{T}} X\right)^{-1} \Sigma\right]+\mu\left(X^{\mathrm{T}} X\right)^{-1} \mu^{\mathrm{T}}\right\} \sigma^{2}=\Lambda \cdot \sigma^{2},
$$

where $\sigma^{2}$ denotes the variance of the error in the population. The input set of DFT structures is identified by $N \times m$ of the correlation functions $X$ including the empty cluster. $\Sigma$ is the $m \times m$ covariance matrix of the correlation functions of the structures in the population and $\mu$ is the $m$-dimensional vec- 
tor of the mean correlation functions of the structures in the population. The distribution of all structures in the population is characterized by $\Sigma$ and $\mu$. Approximate values of $\Sigma$ and $\mu$ can be evaluated for many random configurations within a finite number of atoms. $\Lambda$ can be evaluated using $\Sigma$ and $\mu$ for each set of correlation functions $X$. It should be emphasized that DFT calculations are not required to evaluate $\Lambda$. In the present study, the additional structures are therefore chosen so as to significantly decrease $\Lambda$. We hereafter call these additional structures "probe structures."

\section{B. Computational procedure}

The selections of clusters and probe structures are mutually dependent. Therefore, they should be repeated alternately until the optimal $\mathrm{CE}$ is constructed. In complex systems such as spinels, an iterative procedure based on the selections of clusters and probe structures are quite useful. ${ }^{21}$ First, we prepare initial DFT structures. Here, we consider only binary configurations of divalent and quadrivalent cations on the background of the fcc oxygen sublattice. Using the initial DFT structures, a trial CE that minimizes the CV score is constructed. Here, we use the simulated annealing (SA) for the selection instead of the genetic algorithm. ${ }^{20}$ Then, $N_{\text {probe }}$ probe structures that reduce the coefficient $\Lambda$ by the greatest amount are searched for using the SA. Their DFT energies are calculated. In order to validate the trial CE, we introduce the $\mathrm{CV}$ score for $N+N_{\text {probe }}$ structures with clusters selected from $N$ structures, i.e., $\mathrm{CV}\left(N+N_{\text {probe }}\right)$. If $\mathrm{CV}\left(N+N_{\text {probe }}\right)$ is larger than $\mathrm{CV}(N)$, the trial CE is considered to fail. The probe structures are then added to the input set and a new iterative step is started from the cluster selection. The iterative steps are repeated until $\mathrm{CV}\left(N+N_{\text {probe }}\right)$ and $\mathrm{CV}(N)$ converge. The converged $\mathrm{CV}$ score is regarded as the predictive power of the $\mathrm{CE}$. Details of the iterative procedure have been described in Ref. 21 .

As a result of the iterative calculations, we obtain the total energies of $114,142,128$, and 120 ordered structures for $\mathrm{Mg}_{2} \mathrm{TiO}_{4}, \mathrm{Zn}_{2} \mathrm{TiO}_{4}, \mathrm{Mg}_{2} \mathrm{SnO}_{4}$, and $\mathrm{Zn}_{2} \mathrm{SnO}_{4}$, respectively. We evaluate ECIs with 23, 22, 22, and 22 clusters for $\mathrm{Mg}_{2} \mathrm{TiO}_{4}, \mathrm{Zn}_{2} \mathrm{TiO}_{4}, \mathrm{Mg}_{2} \mathrm{SnO}_{4}$, and $\mathrm{Zn}_{2} \mathrm{SnO}_{4}$, respectively. The clusters are selected from a pool of 126 clusters up to quadruplets. The converged CV scores for four IV-II spinels are less than $7.0 \mathrm{meV} /$ cation.

For the calculation of the total energies, we use the projector augmented-wave method ${ }^{22,23}$ as implemented in the VASP code. ${ }^{24,25}$ The local density approximation ${ }^{26,27}$ is used for the exchange-correlation term. The cut-off energy of the plane-wave-basis set is set at $350 \mathrm{eV}$. The total energies are converged to less than $10^{-2} \mathrm{meV}$. The atomic positions and lattice constants are relaxed until the residual forces become less than $0.01 \mathrm{eV} / \AA$. $k$ points are sampled based on the Monkhorst-Pack scheme ${ }^{28}$ with $2 \times 2 \times 2$ mesh.

Once the optimal CE is constructed, one can predict the cation distribution and thermodynamic properties on the basis of the statistical mechanics. Finite-temperature thermodynamic properties are evaluated using canonical MC simulations with the Metropolis algorithm. ${ }^{29}$ Supercells for the MC simulations are constructed by the $10 \times 10 \times 10$ expansion of

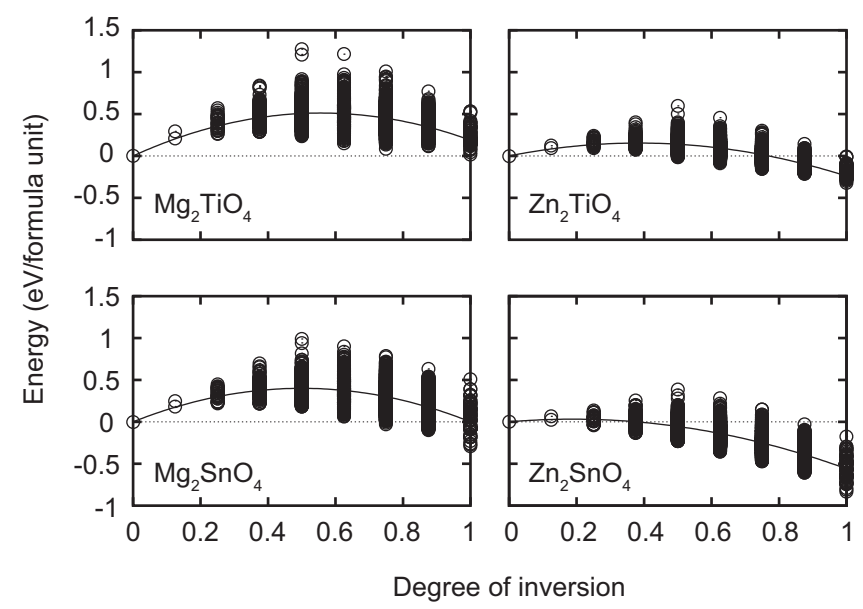

FIG. 2. Predicted internal energies of all configurations within the unit cell. The energies are measured from that of the normal configuration. The solid curves show the fits to a quadratic formula of $\Delta E=a x+b x^{2}$, used in Secs. III C and III D for classifying the ground-state structure and order-disorder behavior in spinel oxides. By fitting the predicted internal energies of all configurations within the unit cell, the energy of the disordered state with $x$ is approximately evaluated.

the unit cell. The MC simulations are performed for over 8000 MC steps per cation to calculate the thermodynamic averages after equilibration. We use the CLUPAN code ${ }^{11,21,30}$ in the series of calculations performed to construct the $\mathrm{CE}$ and to evaluate the finite-temperature properties.

\section{RESULTS AND DISCUSSION}

\section{A. Cation ordering in IV-II spinel oxides}

First, we investigate the cation ordering at zero temperature in four IV-II spinels. In order to search for the cation ordering, we adopt two kinds of procedures: an exhaustive search and an SA. In the exhaustive search, we can find the configuration with the lowest energy directly by calculating the internal energies of all configurations within the unit cell. In the SA, we can find the lowest-energy configuration efficiently by generating successive configurations analogous to the real annealing process. The cation ordering can be searched for in a much larger space of configurations using the SA than the exhaustive search. Figure 2 shows the internal energies of all configurations within the unit cell measured from that of the normal configuration, as a result of the exhaustive search. Three spinels, $\mathrm{Mg}_{2} \mathrm{SnO}_{4}, \mathrm{Zn}_{2} \mathrm{TiO}_{4}$, and $\mathrm{Zn}_{2} \mathrm{SnO}_{4}$, show the lowest energy at the tetragonal inverse configuration with the space group of $P_{4} 22$. The cation ordering of $\mathrm{Zn}_{2} \mathrm{TiO}_{4}$ agrees with that of the experimental lowtemperature structure. For the three spinels, the SA predicts the same cation ordering as predicted from the exhaustive search. Therefore, we reach the conclusion that the cation ordering at zero temperature in the three spinels is $P 4_{1} 22$ tetragonal inverse.

In $\mathrm{Mg}_{2} \mathrm{TiO}_{4}$, the two different procedures predict different configurations. The configuration obtained by the exhaustive search with the lowest energy is the normal one. Meanwhile, 
the SA predicts the cation ordering to be the $P 4_{1} 22$ tetragonal inverse configuration. The $P 4_{1} 22$ inverse configuration has the second lowest energy in the exhaustive search. The energy of the $P 4_{1} 22$ inverse configuration relative to that of the normal configuration is slightly positive by $30 \mathrm{meV} / \mathrm{f}$.u. Therefore, the cation ordering at zero temperature is theoretically the normal spinel in $\mathrm{Mg}_{2} \mathrm{TiO}_{4}$. This contradicts the experimental low-temperature $P 4_{1} 22$ tetragonal inverse structure. It agrees with the cation ordering predicted from the SA. In literature, DFT calculations for 160 configurations including the normal and the $P 4_{1} 22$ inverse configurations have been reported. ${ }^{31}$ The normal and inverse configurations were predicted to be almost equivalent energetically. They claimed that the strong inverse nature in $\mathrm{Mg}_{2} \mathrm{TiO}_{4}$ can be ascribed to the configurational entropy in the inverse state. This interesting cation ordering behavior will be discussed later in this paper.

\section{B. Temperature dependence of cation distribution in IV-II spinel oxides}

Next, we investigate the temperature dependence of the cation distribution. We perform the MC simulations in both a heating process starting from the ground-state structure and a cooling process starting from a configuration equilibrated at a high temperature. Similar to the case of the II-III inverse spinels reported in our previous paper for $\mathrm{MgGa}_{2} \mathrm{O}_{4}$ and $\mathrm{MgIn}_{2} \mathrm{O}_{4},{ }^{12}$ two types of order-disorder behaviors are recognized in $\mathrm{Zn}_{2} \mathrm{TiO}_{4}, \mathrm{Mg}_{2} \mathrm{SnO}_{4}$, and $\mathrm{Zn}_{2} \mathrm{SnO}_{4}$. As temperature increases, the inverse spinels change from the OI state to the DI state, and then from the DI state to the DD state. Calculated temperature dependences of the degree of inversion $x$ in the heating and cooling processes are almost the same although a small hysteresis exists at around the temperature of the order-disorder transition from the OI state to the DI state. The transition from the OI state to the DI state is first-order. The change from the DI state to the DD state is predicted to be nonconvergent.

In order to estimate the temperatures of order-disorder transition from the OI state to the DI state, the MC simulations are performed at a finer interval of $10 \mathrm{~K}$ around the transition temperatures. Because the hysteresis occurs, rigorous evaluation of the transition temperatures involves calculations of the free energies for the OI, DI, and DD states. Alternatively, we estimate the transition temperature from the maximum gap of the temperature dependence of the short-range order (SRO) parameter in the heating process. Calculated temperature dependences of the Warren-Cowley SRO parameter ${ }^{32}$ for the second-nearest octahedraloctahedral pair in the heating process for the three spinels are shown in Fig. 3. Figure 4 shows the order-disorder transition temperatures of inverse IV-II spinels, compared with those of inverse II-III spinels. In $\mathrm{Zn}_{2} \mathrm{TiO}_{4}$, the transition from the OI state to the DI state occurs at around $500 \mathrm{~K}$, which is lower than the experimental transition temperature of $834 \mathrm{~K} .{ }^{15}$ IV-II spinels transform from the OI state to the DI state at higher temperatures than II-III spinels. This can be ascribed to the fact that the change in the Madelung energy upon the exchange of cations in IV-II spinels is larger than that in II-III spinels.

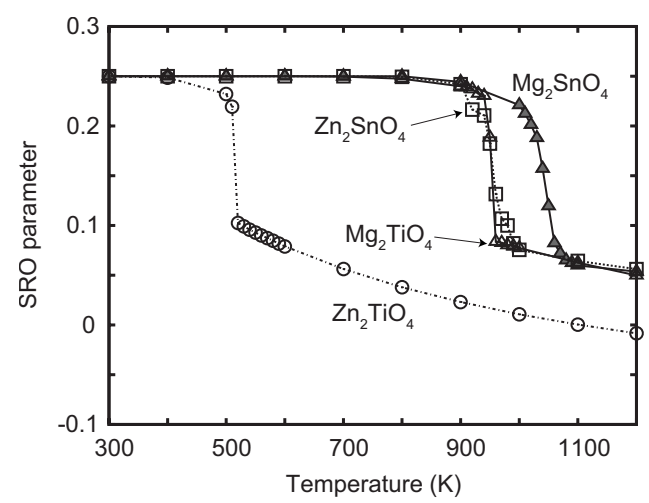

FIG. 3. Temperature dependence of the Warren-Cowley SRO parameter for the second-nearest octahedral-octahedral pair in the heating process. The Warren-Cowley SRO parameter of the pair in $A B_{2} \mathrm{O}_{4}$ spinel is defined as $\left(9\left\langle\varphi_{\text {pair }}\right\rangle-1\right) / 8$, where $\left\langle\varphi_{\text {pair }}\right\rangle$ is the thermal average of the pair-correlation functions.

Contrary to the above three spinels, calculated temperature dependences of the degree of inversion $x$ in the heating and cooling processes are not the same in $\mathrm{Mg}_{2} \mathrm{TiO}_{4}$, as shown in Fig. 5. In the heating process, $\mathrm{Mg}_{2} \mathrm{TiO}_{4}$ changes from the $\mathrm{ON}$ state to the DD state. In the cooling process, $\mathrm{Mg}_{2} \mathrm{TiO}_{4}$ changes from the DD state to the DI state and then from the DI state to the OI state without transforming to the ON state. The origin of the inconsistency of the temperature dependences in the heating and cooling processes will be described later in this paper.

In order to evaluate the temperature of the transition from the OI state, which is metastable at zero temperature, to the DI state in $\mathrm{Mg}_{2} \mathrm{TiO}_{4}$, the $\mathrm{MC}$ simulations are performed in a heating process starting from $P 4_{1} 22$, the OI state. The calculated temperature dependence of the cation distribution for $\mathrm{Mg}_{2} \mathrm{TiO}_{4}$ in the heating process starting from the OI state is shown in Figs. 3 and 4. The phase transition from the OI state to the DI state occurs at around $920 \mathrm{~K}$, which is very close to the experimental values of $933,{ }^{14} 937,{ }^{15}$ and $943-$ $953 \mathrm{~K} \cdot{ }^{33}$

\section{Classification of ground-state structure in spinel oxides}

In order to classify the ground-state structures and orderdisorder behavior in the spinel oxides, a simple model for

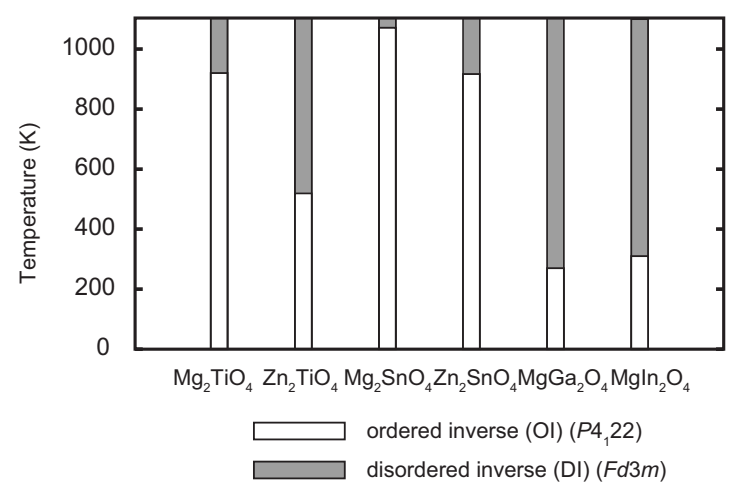

FIG. 4. Predicted temperature dependences of the cation distributions in the heating process from the OI state in IV-II spinels. They are compared with predicted temperature dependences of the cation distributions in $\mathrm{MgGa}_{2} \mathrm{O}_{4}$ and $\mathrm{MgIn}_{2} \mathrm{O}_{4}$ (Ref. 12). 


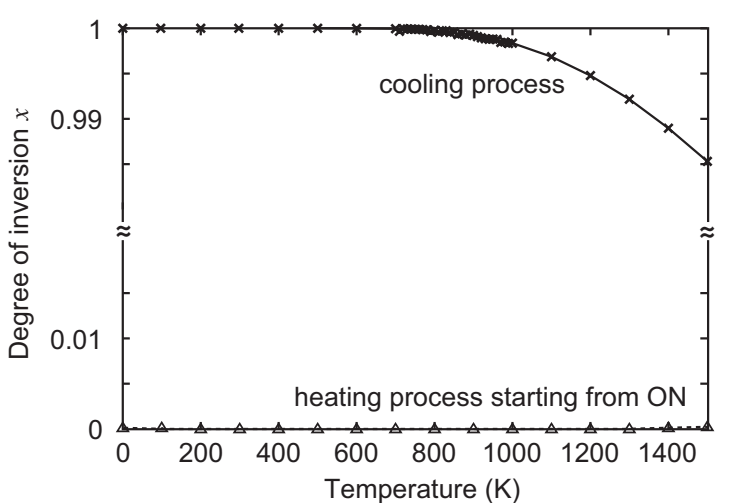

FIG. 5. Temperature dependence of the degree of inversion in $\mathrm{Mg}_{2} \mathrm{TiO}_{4}$ predicted from $\mathrm{MC}$ simulations in the heating process starting from the $\mathrm{ON}$ state and the cooling process starting from a configuration equilibrated at a high temperature.

free energy, proposed in Ref. 34, is used. The internal energy, $\Delta E$, is approximated as a quadratic formula of $x$ expressed as

$$
\Delta E=a x+b x^{2} .
$$

The coefficients $a$ and $b$ can be evaluated from the predicted internal energies of ordered structures. By fitting the predicted internal energies of all configurations within the unit cell by the least-squares technique, we evaluate the internal energy of the disorder state with $x$. The configurational entropy of the disorder state with $x, \Delta S$, per formula unit is approximated as

$$
\begin{aligned}
\Delta S= & -k_{\mathrm{B}}[x \ln x+(1-x) \ln (1-x) \\
& \left.+x \ln \frac{x}{2}+(2-x) \ln \left(1-\frac{x}{2}\right)\right],
\end{aligned}
$$

where $k_{\mathrm{B}}$ denotes the Boltzmann constant. This entropy is derived from the point approximation, where correlations between cations are not considered. The first two terms arise from the degrees of freedom of cations on the tetrahedral sites. The last two terms originate from the degrees of freedom of cations on the octahedral sites. The minimum of the Helmholtz free-energy change, $\Delta F=\Delta E-T \Delta S$, determines the cation distribution at temperature $T$.

In this simple model of free energy, only coefficients $a$ and $b$ control the cation distribution. Figure 6(a) shows a structure map for the cation distribution with respect to $(a, b)$ at zero temperature. The ground-state structures of spinels can be classified into three regions depending on the value of $b / a$ and the sign of $a$. When $b / a>-1$ with positive $a$, the cation distribution is normal. When $b / a<-1$ with positive $a$ or $b / a>-1 / 2$ with negative $a$, the inverse state is the most stable. When $b / a<-1 / 2$ with negative $a$, neither normal nor inverse but an intermediate configuration is stable. Because the inverse state in this model is considered to be the DI state, the area of the inverse state expands on a more rigorous structure map. Sets of values $(a, b)$ obtained by the present calculations for six II-III spinels and four IV-II spinels are shown in Fig. 6(b). The fitted internal energy in IV-II spinels
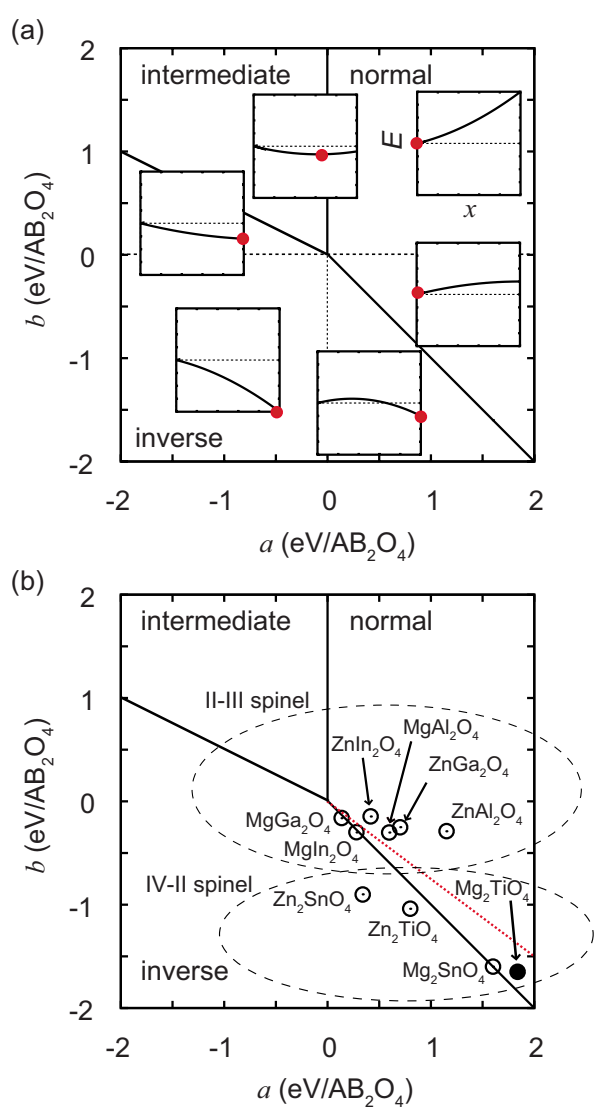

FIG. 6. (Color online) Structure map for the cation distribution with respect to $(a, b)$ at zero temperature. Schematic illustrations of the free energy are shown in (a). The cation distribution that has the minimum free energy is shown by the closed circles. The boundary between the normal and inverse states is the line $b=-a$. The boundary between the inverse and intermediate states is the line $b=-(1 / 2) a$. Sets of values $(a, b)$ obtained by the present calculations for six II-III spinels and four IV-II spinels are shown in (b). The broken line shows the boundary between the general normal spinels and limited spinels which change from the ON state to the DD state through the DI state. This is described as $b=(-3 / 4) a$.

is shown in Fig. 2. In the structure map, $\mathrm{Mg}_{2} \mathrm{SnO}_{4}$ is located on the boundary line between normal and inverse. Strictly speaking, however, because the boundary in Fig. 6(b) is not between the ON and OI states but between the ON and DI states, the stable state of $\mathrm{Mg}_{2} \mathrm{SnO}_{4}$ is the OI state.

\section{Classification of order-disorder behavior in spinel oxides}

The temperature dependence of the cation distribution can also be calculated on the plane $(a, b)$ using the simple model. Because all of the ten spinel oxides have positive $a$ and negative $b$, we focus on the temperature dependence of the cation distribution for the spinels characterized by positive $a$ and negative $b$. In this case, only $b / a$ controls the orderdisorder behavior. Figure 7 shows the dependence of the degree of inversion on normalized temperature $k_{\mathrm{B}} T / a$. As shown also in Fig. 6, the boundary between the normal and inverse states at zero temperature is $b / a=-1$. As temperature increases, the area of the inverse state expands owing to the 


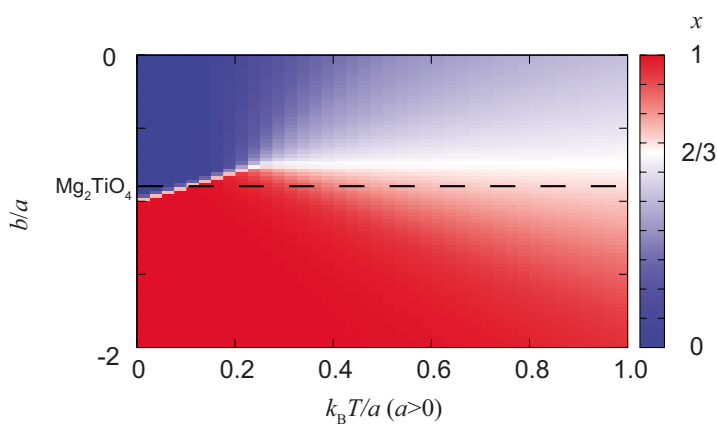

FIG. 7. (Color online) Calculated degree of inversion $x$ as a function of normalized temperature $k_{\mathrm{B}} T / a$. The temperature dependence is determined only by the ratio of the coefficients $b / a$. The normal, inverse, and disorder states are stable in the blue, red, and white regions, respectively.

configurational entropy. The normal spinel far from the boundary changes directly from the ON state to the DD state as temperature increases. It should be noted that a normal spinel near the boundary changes from the ON state to the DI state and then to the DD state as temperature increases.

The derivative of the energy with respect to $x$ at the hightemperature limit of $x=2 / 3$ determines whether the DI state appears in the order-disorder behavior or not. The sign of $d E /\left.d x\right|_{x=2 / 3}$ turns at $b / a=-3 / 4$. Starting from the hightemperature limit, when $b / a>-3 / 4$, the degree of inversion $x$ decreases, i.e., the spinel approaches the ON state, as temperature decreases. On the other hand, when $b / a<-3 / 4$, the degree of inversion $x$ increases, i.e., the spinel approaches the DI state as temperature decreases. Therefore, the normal spinels that have $b / a$ ranging from -1 to $-3 / 4$ with positive $a$ change from the DD state to the ON state through the DI state as temperature decreases. As can be seen in Figs. 6(b) and 7 , only $\mathrm{Mg}_{2} \mathrm{TiO}_{4}$ has $b / a$ ranging from -1 to $-3 / 4$ among six II-III and four IV-II spinel oxides. Thus, the change from the ON state to the DD state through the DI state is expected to occur in limited normal spinels.

\section{E. Order-disorder behavior in $\mathrm{Mg}_{2} \mathrm{TiO}_{4}$}

In $\mathrm{Mg}_{2} \mathrm{TiO}_{4}$, the temperature dependences of the degree of inversion $x$ calculated from MC simulations in the heating and cooling processes are not the same as shown in Sec. III B. The origin of the inconsistency of the temperature dependences may be the fact that intermediate states have high energies, as shown in Fig. 2. In other words, there is a highenergy barrier between the normal and inverse states. As a result, the phase stability remains unclarified.

Performing only standard single atom-flip MC simulations does not provide information about the phase stability between the normal and inverse state separated by the highenergy barrier. The usual approach to examining the stability is to calculate the free energies of both the normal and inverse phases. In a general MC simulation, the configurational free energy is calculated using the thermodynamic integration from reference states with known free energies. The low-temperature limit and the high-temperature limit are generally used for order phases and disorder phases as the reference states, respectively. However, it is difficult to calculate the free energy of normal spinel at high temperatures because the normal state is not obtained by a cooling process starting from the disordered state as shown in Fig. 5. An alternative way to investigate the stability between the phases separated by the high-energy barrier is to deal with both phases simultaneously in one simulation. The multicanonical MC simulation or the entropic-sampling MC simulation can be useful. ${ }^{35-37}$ However, these approaches are anticipated to be very time consuming in a large system.

In the simple free-energy model, $\mathrm{Mg}_{2} \mathrm{TiO}_{4}$ changes from the ON state to the DD state through the DI state as temperature increases. The temperature dependence of the cation distribution in $\mathrm{Mg}_{2} \mathrm{TiO}_{4}$ is shown in Fig. 7. Given the theoretical phase stability, the DI state observed in experiments can be identified as the stable phase. Once the DI state is stabilized, $\mathrm{Mg}_{2} \mathrm{TiO}_{4}$ reaches the metastable OI state during real cooling processes without changing to the $\mathrm{ON}$ state owing to the high-energy barrier between the normal and inverse states. In the SA or the MC simulation, although the generation of successive configurations does not correspond to the real process, some analogy can be made. Therefore, $\mathrm{Mg}_{2} \mathrm{TiO}_{4}$ reaches the metastable OI state in the SA or the MC simulation as well as in experiments.

\section{CONCLUSION}

We have discussed general trends of the ground-state structure and the order-disorder behavior in spinel oxides on the basis of the CE. The ground-state structures of spinels have been classified into three regions depending on two parameters estimated from the CE energies. In inverse spinels, a unique order-disorder behavior has been recognized. The inverse spinels change from the OI state to the DI state and then to the DD state as temperature increases. The normal spinels show different order-disorder behaviors depending on the energy of the inverse state. General normal spinels change directly from the ON state to the DD state as temperatures increases. On the other hand, a limited number of normal spinels such as $\mathrm{Mg}_{2} \mathrm{TiO}_{4}$, in which the energy of the normal state is slightly lower than those of the inverse state, change from the $\mathrm{ON}$ state to the DD state through the DI state. Although the $\mathrm{ON}$ state is the ground state in $\mathrm{Mg}_{2} \mathrm{TiO}_{4}$, once the DI state is stabilized, the metastable OI state is likely to appear in real cooling processes owing to the existence of the high-energy barrier between the normal and inverse states.

\section{ACKNOWLEDGMENTS}

This study was supported by Program for Improvement of Research Environment for Young Researchers from Special Coordination Funds for Promoting Science and Technology (SCF) commissioned by the Ministry of Education, Culture, Sports, Science and Technology (MEXT) of Japan. This study was also supported by Grant-in-Aid for Young Scientists B. 
*seko@cms.mtl.kyoto-u.ac.jp

${ }^{1}$ G. Price, S. Price, and J. Burdett, Phys. Chem. Miner. 8, 69 (1982).

${ }^{2}$ M. Huber, C. R. Acad, Sci., Paris, 244, 2524 (1957).

${ }^{3}$ F. Machatschki, Z. Kristallogr. 82, 348 (1932).

${ }^{4}$ J. E. Weidenborner, N. R. Stemple, and Y. Okaya, Acta Crystallogr. 20, 761 (1966).

${ }^{5}$ H. Schmalzried, Z. Phys. Chem. 28, 203 (1961).

${ }^{6}$ T. F. W. Barth and E. Posnjak, Z. Kristallogr. 82, 325 (1932).

${ }^{7}$ S. H. Wei and S. B. Zhang, Phys. Rev. B 63, 045112 (2001).

${ }^{8}$ J. M. Sanchez, F. Ducastelle, and D. Gratias, Physica A 128, 334 (1984).

${ }^{9}$ D. de Fontaine, Solid State Physics (Academic, New York, 1994), Vol. 47.

${ }^{10}$ F. Ducastelle, Order and Phase Stability in Alloys (Elsevier Science, 1994).

${ }^{11}$ A. Seko, K. Yuge, F. Oba, A. Kuwabara, I. Tanaka, and T. Yamamoto, Phys. Rev. B 73, 094116 (2006).

${ }^{12}$ A. Seko, K. Yuge, F. Oba, A. Kuwabara, and I. Tanaka, Phys. Rev. B 73, 184117 (2006).

${ }^{13}$ F. S. Galasso, Structure and Properties of Inorganic Solids (Pergamon, New York, 1970).

${ }^{14}$ B. A. Wechsler and A. Navrotsky, J. Solid State Chem. 55, 165 (1984).

${ }^{15}$ R. L. Millard, R. C. Peterson, and B. K. Hunter, Am. Mineral. 80, 885 (1995).

${ }^{16}$ H. S. C. O'Neill, S. A. T. Redfern, S. Kesson, and S. Short, Am. Mineral. 88, 860 (2003).

${ }^{17}$ J. W. D. Connolly and A. R. Williams, Phys. Rev. B 27, 5169
(1983).

${ }^{18}$ M. Stone, J. R. Stat. Soc. Ser. B 36, 111 (1974).

${ }^{19}$ A. van de Walle, and G. Ceder, J. Phase, Equilib. 23, 348 (2002).

${ }^{20}$ G. L. W. Hart, V. Blum, M. J. Walorski, and A. Zunger, Nature Mater. 4, 391 (2005).

${ }^{21}$ A. Seko, Y. Koyama, and I. Tanaka, Phys. Rev. B 80, 165122 (2009).

${ }^{22}$ P. E. Blöchl, Phys. Rev. B 50, 17953 (1994).

${ }^{23}$ G. Kresse and D. Joubert, Phys. Rev. B 59, 1758 (1999).

${ }^{24}$ G. Kresse and J. Hafner, Phys. Rev. B 47, 558 (1993).

${ }^{25}$ G. Kresse and J. Furthmüller, Phys. Rev. B 54, 11169 (1996).

${ }^{26}$ D. M. Ceperley and B. J. Alder, Phys. Rev. Lett. 45, 566 (1980).

${ }^{27}$ J. P. Perdew and A. Zunger, Phys. Rev. B 23, 5048 (1981).

${ }^{28}$ H. J. Monkhorst and J. D. Pack, Phys. Rev. B 13, 5188 (1976).

${ }^{29}$ N. Metropolis, A. W. Rosenbluth, M. N. Rosenbluth, A. H. Teller, and E. Teller, J. Chem. Phys. 21, 1087 (1953).

${ }^{30}$ A. Seko, CLUPAN, http://sourceforge.net/projects/clupan (2009).

${ }^{31}$ E. J. Palin, A. M. Walker, and R. J. Harrison, Am. Mineral. 93, 1363 (2008).

${ }^{32}$ J. M. Cowley, J. Appl. Phys. 21, 24 (1950).

${ }^{33}$ F. Oba, A. Seko, H. Hayashi, K. Goto, and I. Tanaka (unpublished).

${ }^{34}$ H. S. C. O’Neill and A. Navrotsky, Am. Mineral. 68, 181 (1983).

${ }^{35}$ B. A. Berg and T. Neuhaus, Phys. Rev. Lett. 68, 9 (1992).

${ }^{36}$ J. Lee, Phys. Rev. Lett. 71, 211 (1993).

${ }^{37}$ F. Wang and D. P. Landau, Phys. Rev. Lett. 86, 2050 (2001). 